\title{
Use of in vivo magnetic resonance spectroscopy for studying metabolic diseases
}

\author{
Jong-Hee Hwang ${ }^{1}$ and Cheol Soo Choi ${ }^{1,2}$
}

Owing to the worldwide obesity epidemic and the sedentary lifestyle in industrialized countries, the number of people with metabolic diseases is explosively increasing. Magnetic resonance spectroscopy (MRS), which is fundamentally similar to magnetic resonance imaging, can detect metabolic changes in vivo noninvasively. With its noninvasive nature, ${ }^{1} \mathrm{H},{ }^{13} \mathrm{C}$ and ${ }^{31} \mathrm{P}$ MRS are being actively utilized in clinical and biomedical metabolic studies to detect lipids and important metabolites without ionizing radiation. ${ }^{1} \mathrm{H}$ MRS can quantify lipid content in liver and muscle and can detect other metabolites, such as 2-hydroxyglutarate, in vivo. Of interest, many studies have indicated that hepatic and intramyocellular lipid content is inversely correlated with insulin sensitivity in humans. Thus, lipid content can be utilized as an in vivo biomarker for detecting early insulin resistance. Employing ${ }^{13} \mathrm{C}$ MRS, hepatic glycogen synthesis and breakdown can be directly detected, whereas ${ }^{31} \mathrm{P}$ MRS provides in vivo adenosine triphosphate (ATP) synthesis rates by saturation transfer methods in addition to ATP content. These in vivo data can be very difficult to assess by other methods and offer a critical piece of metabolic information. To aid the reader in understanding these new methods, fundamentals of MRS are described in this review in addition to promising future applications of MRS and its limitations.

Experimental \& Molecular Medicine (2015) 47, e139; doi:10.1038/emm.2014.101; published online 6 February 2015

\section{INTRODUCTION}

Diabetes mellitus is one of the most common metabolic diseases. According to the World Health Organization (WHO), 347 million people worldwide currently have been diagnosed with diabetes. In industrialized countries with a sedentary lifestyle, the number of diabetic patients is expected to increase at a higher rate. Insulin resistance is a good predictor for the clinical onset of type 2 diabetes mellitus (T2DM) and precedes the disease by many years. To detect early metabolic changes such as developing insulin resistance, noninvasive magnetic resonance (MR) techniques can be an ideal tool for noninvasively monitoring alterations in insulin sensitivity using the lipid content of the liver or muscle as a biomarker in humans and animal models. ${ }^{1-3}$ With the advantage of its noninvasiveness, nuclear magnetic resonance (NMR) has evolved as a powerful medical imaging tool since the development of MR imaging (MRI) in the early 1970s by Lauterbur and Mansfield. ${ }^{4,5}$ In vivo MR spectroscopy (MRS) shares the same fundamentals as MRI and has developed in parallel. It is used to investigate the details of biochemistry and physiology in metabolic diseases in addition to the morphological information assessed by MRI.

To examine metabolic disorders, many clinical and biomedical studies have been conducted using MRS methods, for example, the quantification of hepatic lipid and glycogen content as well as tricarboxylic acid (TCA) cycle or adenosine triphosphate (ATP) synthesis rates in vivo in real time using ${ }^{1} \mathrm{H},{ }^{31} \mathrm{P}$ and ${ }^{13} \mathrm{C}$ MRS. These data are difficult to assess by other modalities. Together these noninvasive methods can provide crucial information for metabolic studies and clinical research. For clinical studies, the noninvasiveness is a prime advantage of the methods; however, for preclinical research, the ability to perform longitudinal studies without killing animals adds value due to the high cost of transgenic and knockout mice. Thus, this noninvasive approach also helps to reduce the number of animals being killed and to eliminate interanimal variances by longitudinal follow-up of the same animal.

In this review, the most commonly used and promising MRS methods are described, including ${ }^{1} \mathrm{H},{ }^{31} \mathrm{P}$ and ${ }^{13} \mathrm{C} \mathrm{MRS}$, with a particular emphasis on metabolic diseases.

\footnotetext{
${ }^{1}$ Korea Mouse Metabolic Phenotyping Center (KMMPC), Lee Gil Ya Cancer and Diabetes Institute, Gachon University, Incheon, Korea and ${ }^{2}$ Division of Endocrinology, Department of Internal Medicine, Gachon University Gil Medical Center, Incheon, Korea

Correspondence: Professor J-H Hwang or Dr CS Choi, Department of Molecular Medicine, Lee Gil Ya Cancer and Diabetes Institute, Gachon University, Yeonsu-Gu, Songdo-Dong 7-45, Incheon 406-840, Korea. E-mail: jonghhwang@gachon.ac.kr or cschoi@gachon.ac.kr Received 14 October 2014; accepted 29 October 2014
} 
a

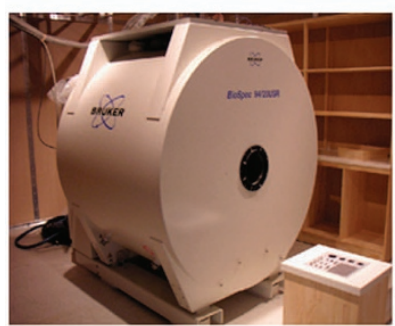

b
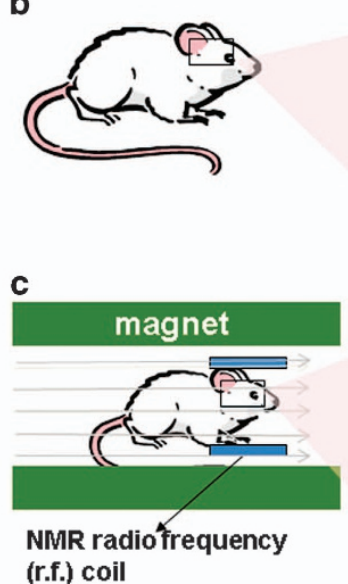

d

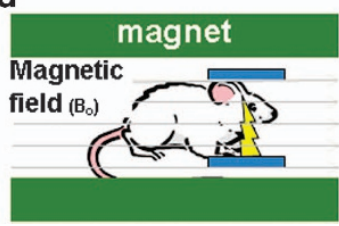

Without magnetic field

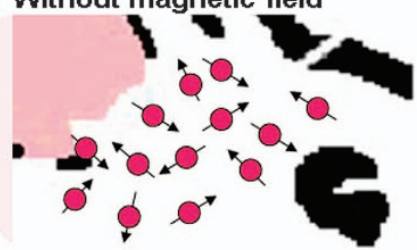

With magnetic field
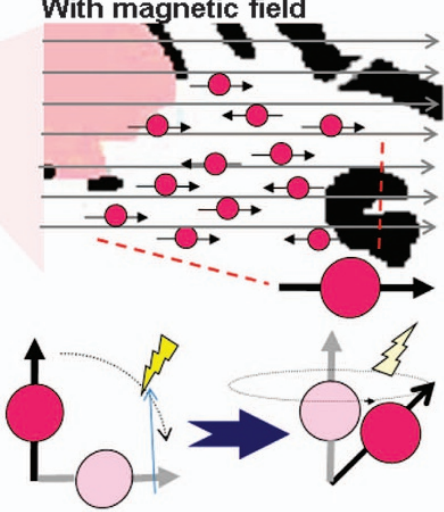

RF Energy by a coil Energy emitted

Figure 1 (a) Bruker 9.4T magnetic resonance spectrometer in Lee Gil Ya Cancer and Diabetes Institute, Gachon University. (b, c) Without a magnetic field (outside of the magnet), all of the spins are randomly oriented (b), but within a field (inside of the magnet), all of the spins are aligned with or against the field (quantized at different energy levels, c). When all of the spins are combined, a longitudinal magnetization is generated parallel to the magnetic field. (d) When a small amount of energy is applied to the magnetization by an radiofrequency (r.f.) coil, the magnetization is flipped to another orientation. However, the magnetization tries to return to its original position (the thermal equilibrium (c)) with precession. While in precession, the magnetization also emits energy (d). This energy is detected by a receiver, which generates nuclear magnetic resonance signals.

\section{GENERAL OVERVIEW OF MRI AND MRS: SIMILARITIES AND DIFFERENCES}

MRI and MRS data can be obtained using a spectrometer, as shown in Figure 1a, or MRI scanners in hospitals. Among all nuclei, only nonzero nuclear spins $(I \neq 0)$ are detectable by NMR, for example, ${ }^{1} \mathrm{H},{ }^{31} \mathrm{P}$ and ${ }^{13} \mathrm{C}$. As shown in Figure $1 \mathrm{~b}$, without a magnetic field (when an animal is outside the range of a magnet), all of the spins are randomly oriented. However, within a magnetic field (inside of a magnet, Figure 1c), all of the spins are aligned with or against the field. In other words, nuclear spin energy levels are quantified at different levels. When the magnetic fields of all spins are summed, a net longitudinal magnetization is generated parallel to the magnetic field, as shown in Figure 1c.

A radiofrequency coil is a special device that can emit energy and receive signals. A small amount of energy is applied to this net magnetization, causing the magnetization to flip to another orientation, as demonstrated in Figure 1d. However, as soon as this transition occurs, the magnetization tries to return to its original orientation (the thermal equilibrium state as in Figure 1c) with precession. During this precession, a small amount of energy is emitted and is detected by a receiver. This produces NMR signals, which eventually generate MRS and MRI after Fourier transformation.

Even though MRI and MRS are fundamentally similar, in contrast to MRI the major advantage of MRS originates from chemical shift information, which is another dimension that expresses the degree of shielding by neighboring electrons of a nuclei within one molecule. For example, protons attached to the $\mathrm{C} 2$ vs the $\mathrm{C} 1$ carbon in ethanol $\left(\mathrm{C}_{(2)} \mathrm{H}_{3}-\mathrm{C}_{(1)} \mathrm{H}_{2}-\mathrm{OH}\right)$ will have different chemical shifts, as demonstrated in Figure $2 \mathrm{~b}$. Thus, the two different types of protons in ethanol can be clearly distinguished in MRS. In contrast, because $70-90 \%$ of living tissues are composed of water, a normal MRI is an image of water that is taken at one chemical shift of water protons at 4.7 parts per million (p.p.m.; Figure 2a). The anatomical structure of an object in MRI is determined by two to three different spatial dimensions, while MRS methods bring in another dimension of data by providing biochemical information of molecules (metabolites) in living systems via chemical shifts.

\section{MRI and MRS: their similarities and differences}

Proton $\left({ }^{1} \mathrm{H}\right)$ MRI and MRS are the most widely utilized methods in metabolic diseases because the signal-to-noise ratio (SNR) of proton MRS/MRI is generally higher than other nuclei. Although the cost for ${ }^{1} \mathrm{H}$ MRS(/MRI) examinations is higher than computed tomography and ultrasound, this modality is widely used due to the accurate assessment of anatomy and metabolites, such as liver lipids expressed as a percentage by ${ }^{1} \mathrm{H}$ MRS.

As previously mentioned, MRI and MRS share a common physical background. MRI is an imaging technique employing NMR, while MRS also uses NMR, but is spectroscopy. 
a

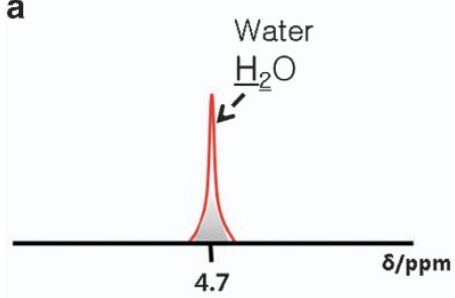

b

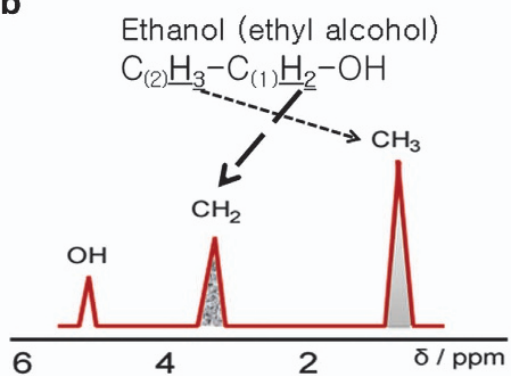

Figure 2 (a) A NMR spectrum of water $\left(\mathrm{H}_{2} \mathrm{O}\right)$. Water protons resonate at $\sim 4.7$ p.p.m. in living tissues. (b) Chemical structure of ethanol and its simplified NMR spectrum. Different types of protons (attached to $\mathrm{C} 1$ and $\mathrm{C} 2$ carbons) are resonated at two different chemical shifts. Therefore, each different proton can be distinguished in magnetic resonance spectroscopy. NMR, nuclear magnetic resonance.

MRS can offer a quantitative assessment of various metabolites and lipids in living tissue via chemical shift differences of each metabolite that cannot be separately detected by MRI. As demonstrated in Figure 3a (the upper row), MRS data are acquired in a similar fashion as MRI on an MR scanner, but the resulting spectra can show each different metabolite at different chemical shifts from a selected voxel. In the lower row (Figure 3b), MRI provides several brain slice images with various image contrasts depending on employed pulse sequences and parameters. Therefore, MRI has great strength to reveal anatomical structure, while MRS offers biochemical information about the living tissue. When the two methods are combined, biochemical data of a specific location with morphological information can be assessed. Thus, these techniques provide important information for medicine and its related research.

\section{${ }^{1} \mathrm{H}$ MRS}

\section{${ }^{1} \mathrm{H}$ MRS for hepatic lipid quantification}

Currently, hepatic lipid content by ${ }^{1} \mathrm{H}$ MRS is the gold standard measurement and is often utilized for clinical trials to develop new hepatic lipid-lowering drugs and employed in preclinical studies. This technique has received attention in the medical community particularly due to several pragmatic applications. Liver and intracellular muscular lipid content reportedly correlate with insulin resistance, which is the best predictor for the clinical onset of T2DM. Thus, lipid content, as assessed by ${ }^{1} \mathrm{H}$ MRS, can serve as a surrogate in vivo marker for insulin sensitivity. The principle of this method is simple. As demonstrated in Figure 4b, separate water and lipid signals

are easily detected and quantified by ${ }^{1} \mathrm{H}$ MRS. Lipid signals can be converted to percent (\%) lipid relative to water. MRI is also used in addition to this ${ }^{1} \mathrm{H}$ MRS method to quantify adipose tissue volume such as visceral adipose tissue (sagittal view (Figure 4c) and transverse view (Figure 4d)), which cannot be easily assessed by other imaging methods, for example, dual-energy X-ray absorptiometry.

\section{${ }^{1} \mathrm{H}$ MRS for intramyocellular lipids}

Another major advantage of muscular lipid measurements by ${ }^{1} \mathrm{H}$ MRS is the intramyocellular (IMCL) and extramyocellular (EMCL) lipid quantitation without the manual cleaning of EMCL adipose tissue. This separation of the two components is achieved by a bulk magnetic susceptibility effect in soleus and tibialis anterior muscles (Figures $5 \mathrm{a}$ and $\mathrm{b}$ ), resulting in distinct chemical shifts between IMCL and EMCL due to the different characteristics of fat states, that is, IMCL lipids have a spherical shape (like a lipid droplet), and EMCL lipids form in fiber-like bundles (like a semisolid), which show a strong orientation in parallel with the muscle fibers. ${ }^{6}$ Thus, this modality provides not only a noninvasive approach, but it also removes potential quantification errors that originated from incomplete manual EMCL cleaning. Numerous studies have demonstrated a correlation of hepatic lipid content and IMCL with insulin resistance. ${ }^{1-3,7}$ Furthermore, IMCL assessment by ${ }^{1} \mathrm{H}$ MRS can also be utilized as an indicator of muscular lipid metabolism during exercise. ${ }^{8}$

\section{Compositional analysis of fat by ${ }^{1} \mathrm{H}$ MRS}

Recently, fat quantity and composition are receiving attention because of their relevance to metabolism and cardiac diseases. Some previous publications noted that saturated and unsaturated fats can have a different impact on metabolism ${ }^{9}$ and are potentially related to cardiac disorders. ${ }^{10-12}$ Thus, noninvasive assessments of fat composition by MRS could also become a powerful tool in relevant research. In tissues where lipid concentrations are very high, such as in bone marrow, fat compositional analysis by ${ }^{1} \mathrm{H}$ MRS can be powerful. ${ }^{13-15}$ More discussion on this topic will be described in the ${ }^{13} \mathrm{C}$ MRS section.

\section{${ }^{31} \mathrm{P}$ MRS}

Overview of ${ }^{31} \mathrm{P}$ MRS

Historically, ${ }^{31} \mathrm{P}$ MRS has been utilized for the detection of ATP and phosphocreatine (PCr) as early as MRI, in the 1970s. ${ }^{16-18}$ Next to protons, ${ }^{31} \mathrm{P}$ nuclei demonstrate a high SNR due to the $99 \%$ natural abundance of the ${ }^{31} \mathrm{P}$ isotope. Rates of ATP synthase and forward creatine kinase activity can be assessed in humans and animals using magnetization transfer ${ }^{31} \mathrm{P}$ MRS. Recently, ATP and other phosphorus metabolite quantities have been related to metabolic alterations, such as those that occur in insulin-resistant T2DM. ${ }^{19}$ Moreover, using saturation transfer ${ }^{31} \mathrm{P}$ MRS, ATP synthesis rates were reportedly reduced in muscles from the elderly and in the offspring of T2DM patients. ${ }^{20,21}$ Thus, both absolute phosphorus metabolite quantities and kinetic information are 


\section{Magnetic Resonance Spectroscopy (MRS) vs. Magnetic Resonance Imaging (MRI)}

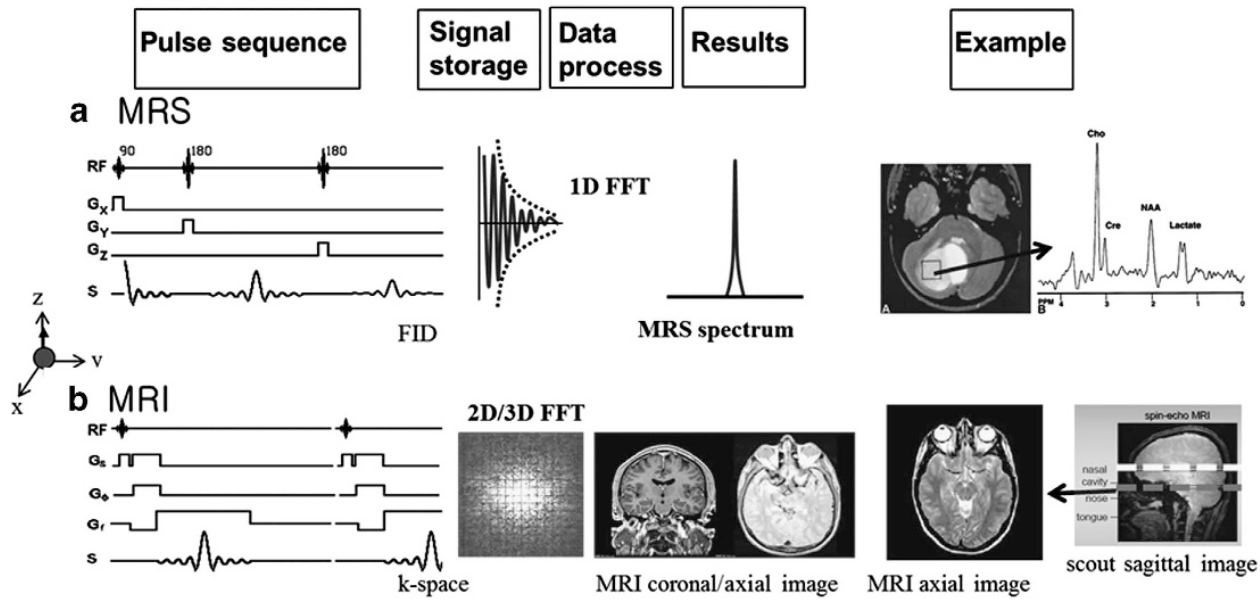

Figure 3 Comparison between MRS and MRI. (a) For MRS, a schematic explanation on how to obtain an MR spectrum and its example in the human brain. Time domain data (free induction decay: FID) were acquired and they were converted to frequency domain data through fast Fourier transformation (FFT). The resulting spectrum demonstrated a peak/peaks as shown in the example of a tumor. Within the tumor, choline (Cho), creatine (Cre), NAA(N-acetyl aspartate) and lactate doublet peaks are clearly demonstrated. (b) For MRI, a schematic explanation for how to obtain an MRI is provided. 2D/3D time domain data were converted to 2D/3D spatial domain data via FFT. The resulting images can exhibit T1- or T2-weighted contrast depending on the sequence and the parameters employed. MRI, magnetic resonance imaging; MRS, magnetic resonance spectroscopy; 2D, two dimensional; 3D, three dimensional.

a

b

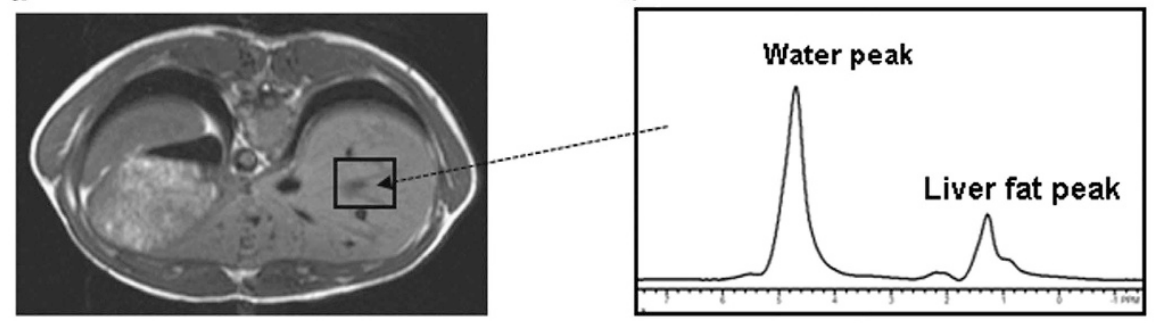

Axial image of liver

${ }^{1} \mathrm{H}$ MRS of selected voxel in the liver

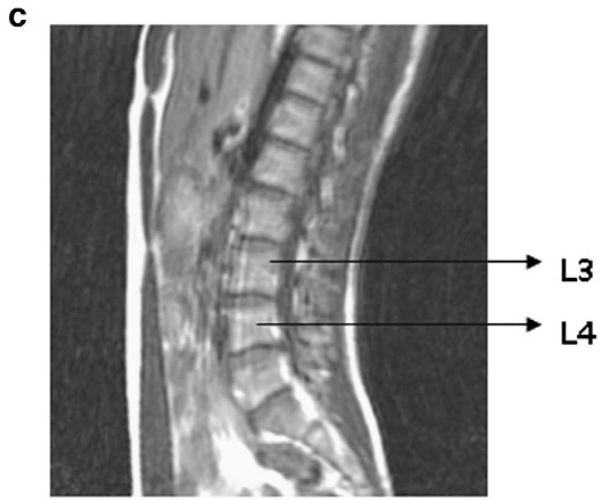

Sagittal image of torso d

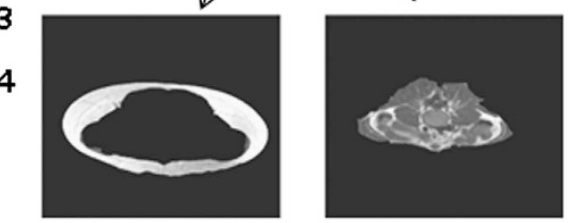

\section{Separating subcutaneous fat from other areas}

Figure 4 (a) Axial image of the liver and the voxel where spectroscopic measurements were obtained. (b) ${ }^{1} \mathrm{H}$-magnetic resonance (MR) spectrum from the liver clearly shows the methylene peak of triglycerides. (c) Sagittal image of an abdomen; the lumbar spine was set as a landmark to select the location for subcutaneous and visceral fat analysis in transverse images. Lumbar spine L3 and L4 are denoted. (d) Transverse image of an abdomen for fat analysis. Subcutaneous and visceral fat were separated (bottom) and the fat content was quantified after converting those images to black-and-white binary images. ${ }^{1}$ 
useful to examine changes in metabolism, aging and diseases by assessing enzyme activities and mitochondrial function. ${ }^{19-24}$ ${ }^{31} \mathrm{P}$ MRS has also been utilized in animal models to evaluate the effect of diets on animal models. ${ }^{24,25}$ Another key player in energy metabolism, nicotinamide adenine dinucleotide, can also be detected and analyzed using ${ }^{31} \mathrm{P}$ MRS. ${ }^{26}$

Quantification of phosphorus metabolites and their role as biomarkers

In ${ }^{31} \mathrm{P}$ MRS, many phosphorus metabolites can be detected, including ATP, PCr, phosphocholine, glycerophosphocholine, glucose-6-phospahte (G6P) and others. ${ }^{27}$ A family of phosphomonoesters was detected at $\sim 6.4-6.6$ p.p.m. in Figure 6 b, which includes G6P. G6P is an important intermediate for glucose oxidation. There is a defect in glycogen synthesis in muscles of T2DM patients. ${ }^{28}$ To find the mechanism(s) behind the defect, Shulman et al measured G6P and found lower G6P concentrations in muscles of T2D patients using ${ }^{31} \mathrm{P}$ MRS. From the data, they concluded that there was a defect in glucose transport and/or phosphorylation in T2DM patients. ${ }^{29}$ Later, a problem in glucose transport was detected in T2DM using ${ }^{31} \mathrm{P}$ MRS with additional studies. ${ }^{30}$

Traditionally, phosphorus metabolites have been well utilized to examine myopathy. For example, Barnes et al. ${ }^{31}$

a

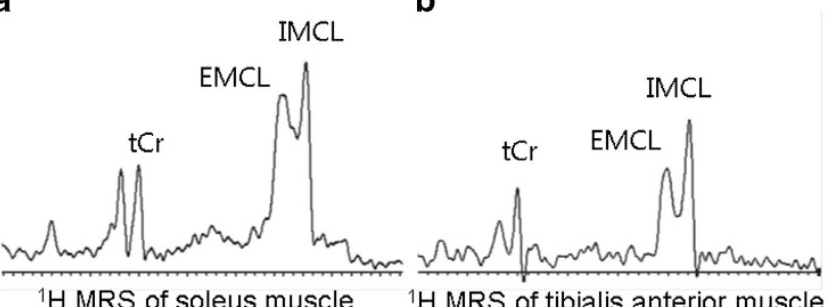

Figure 5 (a) ${ }^{1} \mathrm{H}$-MR spectrum from soleus muscle. (b) ${ }^{1} \mathrm{H}$-spectrum from tibialis anterior muscle. Intramyocellular lipid (IMCL), total creatine ( $\mathrm{tCr}$ ) and extramyocellular lipid (EMCL) peaks are delineated. ${ }^{1}$ observed increased $\mathrm{Pi}$, a reduced $\mathrm{PCr} / \mathrm{ATP}$ ratio and elevated $\mathrm{pH}$ in muscles of patients with myotonic dystrophy, and Kemp et al. ${ }^{32}$ reported mitochondrial dysfunction in muscles using ${ }^{31} \mathrm{P}$ MRS combined with exercise.

\section{Magnetization transfer ${ }^{31} \mathrm{P}$ MRS experiment for ATP synthase and forward PCr kinase activity}

Elegant ${ }^{31} \mathrm{P}$ MRS magnetization transfer experiments were utilized to assess kinetic information in humans and animals. A series of ${ }^{31} \mathrm{P}$ studies was conducted to extract ATP synthase rates in animals. ${ }^{25} \mathrm{~A}$ similar study was performed in the elderly and the muscle ATP synthesis rates were slightly reduced compared with the younger subject group. ${ }^{21}$ Many other studies utilized saturation transfer ${ }^{31} \mathrm{P}$ MRS techniques to study the mitochondrial function as mentioned before. ${ }^{20-25}$ Furthermore, another ${ }^{31} \mathrm{P}$ magnetization transfer technique can assess PCr kinase activity in vivo. This method has been utilized to investigate high-energy phosphate metabolism for skeletal and cardiac muscles as well as the brain in normal and pathologic cases. ${ }^{33-35}$

\section{CONVENTIONAL ${ }^{13} \mathrm{C}$ MRS AND HYPERPOLARIZED ${ }^{13} \mathrm{C}$ DETECTION}

\section{General overview of ${ }^{13} \mathrm{C}$ MRS}

The stable carbon isotope ${ }^{13} \mathrm{C}$ is NMR detectible but naturally occurs at only $1.1 \%$ among all carbon isotopes. Thus, the intrinsic SNR for ${ }^{13} \mathrm{C}$ is much lower than ${ }^{1} \mathrm{H}$ and ${ }^{31} \mathrm{P}$ MRS. However, ${ }^{13} \mathrm{C}$ MRS has a wide chemical shift range, which makes many different carbons easily distinguishable. This characteristic adds to the value of ${ }^{13} \mathrm{C}$ MRS, such as for glycogen detection. In ${ }^{1} \mathrm{H}$ MRS, the differentiation of protons attached to C-1 glucose and C-1 glycogen is very challenging due to the proximity of the two signals and overlapping nearby strong water signals. However, in ${ }^{13} \mathrm{C}$ MRS, the separate detection of C-1 carbons in a glucosyl unit in glycogen and glucose are detectible due to the wide range of chemical shifts (Figure 7a). Moreover, the time course change in hepatic a

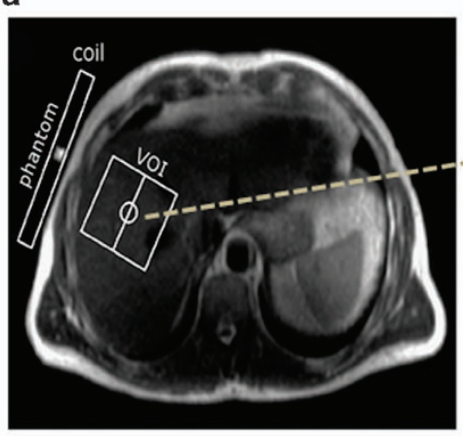

Transverse MRI around the liver b

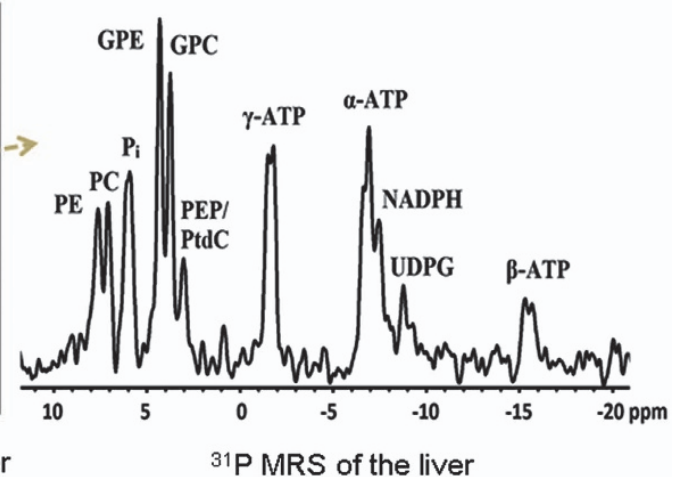

Figure 6 (a) ${ }^{31} \mathrm{P}$ MRS of human liver. Selected voxel in the transverse image around the liver is shown with the r.f. coil position and the reference phantom for absolute phosphorus metabolite quantification. (b) The human ${ }^{31 P} \mathrm{MRS}$ of the liver is shown in the right panel. Hepatic phosphorus metabolites are $\alpha$-, $\beta$ - and $\gamma$-adenosine triphosphate (ATP), uridine diphosphoglucose (UDPG), nicotinamide adenine dinucleotide (/phosphate) (NADP(/H)), phosphatidylcholine (PtdC), inorganic phosphate (Pi), phosphocholine (PC), phosphoethanol (PE), glycerophosphoethanolamine (GPE) and glycerophosphocholine (GPC). MRS, magnetic resonance spectroscopy; r.f., radiofrequency. ${ }^{27}$ 
glycogen was clearly depicted in Figure $7 \mathrm{~b}$ without any exogenous labeling.

Due to low endogenous natural abundance of ${ }^{13} \mathrm{C}$, an exogenously labeled ${ }^{13} \mathrm{C}$ signal can be easily detectible, enabling pulse-chase-type experiments to be performed. However, without exogenous labeling, ${ }^{13} \mathrm{C}$ MRS struggles with low SNR. Recently, to overcome low SNR, hyperpolarizing methods have been introduced and demonstrate that SNR enhancement can be achieved by $10^{3}-10^{4}$ times in a few selected metabolites. This method potentially will resolve the low SNR issue and contribute to a further broadening of ${ }^{13} \mathrm{C}$ MRS usage. This will be discussed more in the hyperpolarized ${ }^{13} \mathrm{C}$ MRS section.

\section{${ }^{13} \mathrm{C}$ MRS for glycogen detection}

As C-1 liver and muscle glycogen is detectible, this method is utilized to quantify the muscular and hepatic glycogen content during nutrient ingestion or exercise in metabolic studies. Because hepatic glycogen concentration is reasonably high (300-400 mmoles of glucosyl unit per liter of tissue in humans), this method can be performed by either natural abundance or exogenous ${ }^{13} \mathrm{C}$ labeling. Using this method as described in Figures $7 \mathrm{a}$ and $\mathrm{b}$, glycogen breakdown and synthesis rates were determined, and altered glycogen metabolism in T1D was identified. ${ }^{36,37}$ Glycogen storage disease can also be noninvasively identified using the same method. ${ }^{38}$ Furthermore, combining these studies with ${ }^{13} \mathrm{C}$ glucose

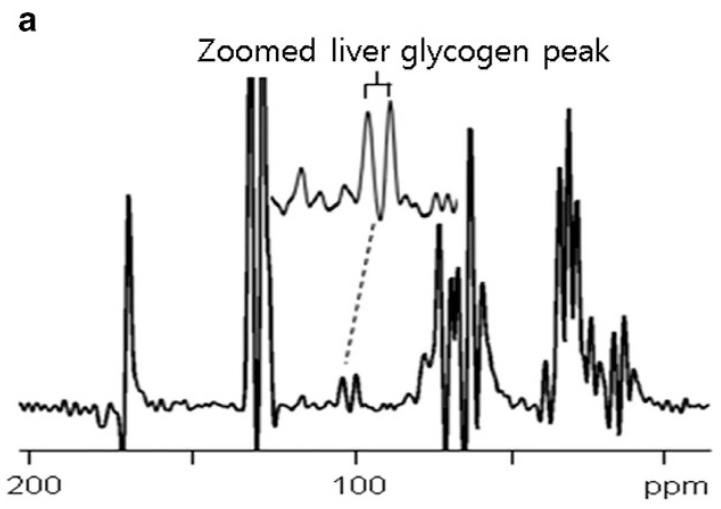

\section{b}
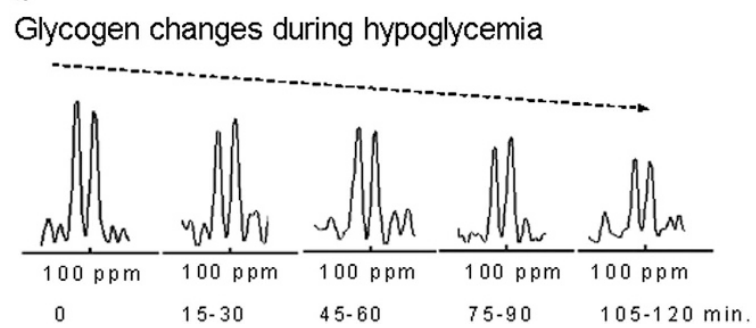

Figure $7{ }^{13} \mathrm{C}$ NMR spectra from the liver. (a) C-1 glycogen of a doublet peak at 100.1 p.p.m. clearly exhibited together with the lipid peaks from the hepatic lipid carbons. The inlayed trace is zoomed clearly demonstrating the C-1 glycogen doublet. (b) The time course spectra of glycogen peaks at 100.1 p.p.m. demonstrate a clear reduction of $\mathrm{C}-1$ glycogen during a hypoglycemic clamp on a control subject. NMR, nuclear magnetic resonance. ${ }^{37}$ labeling, further kinetic studies were conducted to estimate liver glycogen rates. ${ }^{39}$

\section{Compositional lipid analysis}

By taking advantage of the wide spectral range, many carbon signals in lipids also can be separately detected using ${ }^{13} \mathrm{C}$ MRS. ${ }^{40}$ As demonstrated in previous publications, the effects on body fat composition by different types of diets were studied using natural abundance ${ }^{13} \mathrm{C}$ MRS. ${ }^{40}$ Differences of each diet were clearly detected: fish oil (polyunsaturated fatty acid; Figure 8a), normal diet (Figure 8b) and Lorenzo oil (Figure 8c). Thus, ${ }^{13} \mathrm{C}$ MRS can be utilized for noninvasively monitoring dietary effects.

\section{${ }^{13} \mathrm{C}$ MRS for TCA cycle rates}

Using exogenous labeled substrates such as $\mathrm{C} 1$ glucose and $\mathrm{C} 1$ acetate, labeled intermediates in the TCA cycle or relevant intermediates such as glutamate/glutamine can be measured by ${ }^{13} \mathrm{C}$ MRS, and TCA cycle rates and other important fluxes can be assessed ${ }^{41}$ Moreover, by combining saturated transfer ${ }^{31} \mathrm{P}$ MRS and TCA cycling, muscle oxidative capacity was reduced in the offspring of T2D compared with controls. ${ }^{20,21}$

\section{Hyperpolarized ${ }^{13} \mathrm{C}$ MRS}

Despite many advantages of ${ }^{13} \mathrm{C}$ MRS, this method suffers from low SNR due to its low natural abundance (1.1\%) and low gyromagnetic ratio. Thus, increasing SNR is the prime goal for

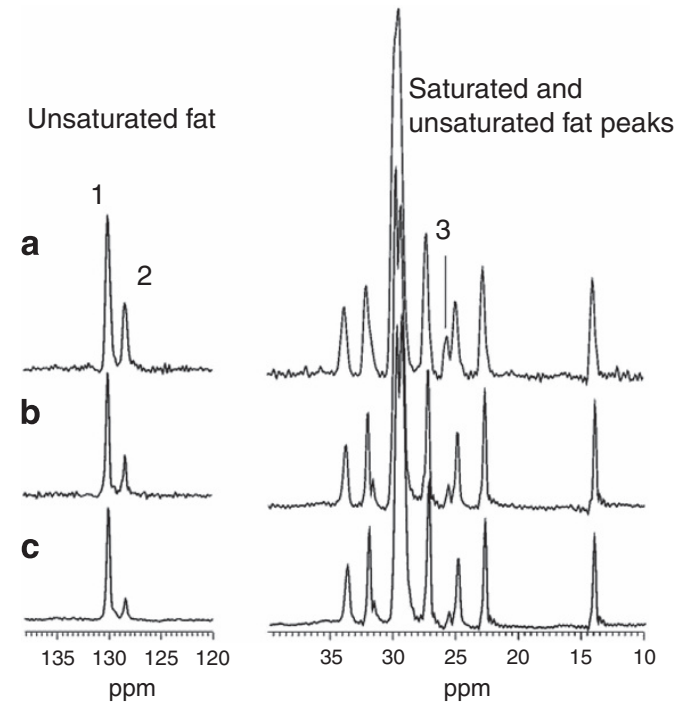

Figure 8 Dietary effect of PUFA and MUFA diets. The differences in PUFA levels are clearly delineated in peak 2 (double-bond peaks in PUFA) and 3 (methylene peaks in between two double bonds in PUFA) in response to the diets. (a) Spectrum from a subject on PUFA (fish oil) diet for $>7$ years. (b) Spectrum from a control subject who was not on any special diet. (c) Spectrum from a subject on a MUFA (Lorenzo's oil) diet for 8 years ${ }^{40}$. All of the PUFA peaks ( 2 and 3 peaks) were increased in a subject that had been on a fish oil diet compared with normal and MUFA diets with reference to peak 1 (all double-bond peaks, including MUFA). MUFA, monounsaturated fatty acid; PUFA, polyunsaturated fatty acid. ${ }^{40}$ 
${ }^{13} \mathrm{C}$ MRS users. By hyperpolarizing ${ }^{13} \mathrm{C}$ spin distribution, the SNR of ${ }^{13} \mathrm{C}$ MRS can be enhanced on the order of $10^{3}-10^{4}$. With recent technical advances in nuclear dynamic polarizers, applications of hyperpolarized ${ }^{13} \mathrm{C}$ MRS have been further demonstrated in animal models, particularly in cancer research due to rapid turnover to lactate with an injection of hyperpolarized C-1 pyruvate. There are two different approaches to hyperpolarize ${ }^{13} \mathrm{C}$ nuclei: one approach dynamically polarizes ${ }^{13} \mathrm{C}$ using a dynamic nuclear polarizer and the other approach utilizes chemically induced parahydrogens. Currently, most published in vivo studies have utilized a dynamic nuclear polarizer approach. In brief, this process artificially increases excitable ${ }^{13} \mathrm{C}$ spins by $10^{3}-10^{4}$ times; however, the polarized spins are unstable and will relax back to the thermal equilibrium state. Thus, all MR examinations with hyperpolarized substrates have to compete within an order of the T1 relaxation times to fully exploit the advantage of this method. Because tumor cells predominantly choose anaerobic processes, the most recent publications using hyperpolarized techniques described detecting anaerobic cycle products such as lactate. Because metabolic diseases do not normally demonstrate high concentrations of anaerobic products, aerobic products involving the TCA cycle are of more importance. A recent publication demonstrated that 2-oxyglutarate in the TCA cycle was detected using hyperpolarized pyruvate ${ }^{42}$ which is the first evidence of utilization of this method in aerobic processes. In the future, the utility of this method might increase outside of oncology applications.

\section{Other metabolic biomarkers using ${ }^{1} \mathrm{H}$ and ${ }^{13} \mathrm{C}$ MRS}

As mentioned before, traditional ${ }^{1} \mathrm{H}$ MRS has been used for various applications, for example, central nervous system diseases, cancer and metabolic diseases. Recently, as a new tumor biomarker, the detection of 2-hydroxyglutarate has been reported by the elegant optimization of ${ }^{1} \mathrm{H}$ MRS. ${ }^{43}$ Mutations in isocitrate dehydrogenases 1 and 2 relate to certain types of gliomas. Indeed, elevated 2-hydroxyglutarate was detected by ${ }^{1} \mathrm{H}$ MRS in gliomas with isocitrate dehydrogenase mutations. ${ }^{43}$ Thus, one can further utilize ${ }^{1} \mathrm{H}\left(\beta^{31} \mathrm{P} /{ }^{13} \mathrm{C}\right)$ MRS in the future for detecting specific biomarkers based on unusual enzymatic activities in diseases.

\section{LIMITATIONS AND OUTLOOK}

In addition to its noninvasive nature, ${ }^{1} \mathrm{H},{ }^{31} \mathrm{P}$ and ${ }^{13} \mathrm{C}$ MRS methods have provided unique biochemical information that would be difficult to obtain by other methods. It is anticipated that these modalities will serve to develop more complete phenotypic data in humans and animal models, which will become crucial for personalized medicine in the future. Although MRS is costly compared with other imaging modalities, it has found its specific role in metabolic research due to its biochemical specificity. Furthermore, this method can also detect biochemical intermediates, which can connect biochemistry with systemic physiology, for example, G6P, 2-hydroxyglutarate.
The limitation of this method is that it is often not easy to provide standardized protocols in a clinical setting or animal model studies compared with MRI. These modalities also often require two-channel scanners for proton decoupling, whereas routine clinical MRI scanners are equipped with only one channel.

Moreover, due to low sensitivity, the acquisition time tends to be longer compared with MRI. However, with further technical development in hardware and software, the required time for these methods can be shortened in the future. New specific biomarkers for a disease/metabolic change can also be developed to extend the applications of MRS methods. Furthermore, effective combinations of these MRS methods with other modalities may bring more comprehensive and powerful examinations of metabolic changes.

\section{CONFLICT OF INTEREST}

The authors declare no conflict of interest.

\section{ACKNOWLEDGEMENTS}

This work was supported by the Bio \& Medical Technology Development Program of the National Research Foundation (NRF) funded by the Ministry of Science, ICT \& Future Planning (2013M3A9D5072563), the Korea Healthcare Technology R\&D Project, Ministry for Health, Welfare \& Family Affairs, Korea (A102060) and the Gachon University Gil Medical Center (Grant \#2013-33).

1 Hwang J-H, Stein DT, Barzilai N, Cui MH, Tonelli J, Kishore $\mathrm{P}$ et al. Increased intrahepatic triglyceride is associated with peripheral insulin resistance: in vivo MR imaging and spectroscopy studies. Am J Physiol Endocrinol Metab 2007; 293: E1663-E1669.

2 Szczepaniak LS, Babcock EE, Schick F, Dobbins RL, Garg A, Burns DK et al. Measurement of intracellular triglyceride stores by $\mathrm{H}$ spectroscopy: validation in vivo. Am J Physiol Endocrinol Metab 1999; 276: E977-E989.

3 Yki-Järvinen H. Liver fat in the pathogenesis of insulin resistance and type 2 diabetes. Dig Dis 2010; 28: 203-209.

4 Lauterbur PC. Image formation by induced local interaction: examples employing nuclear magnetic resonance. Nature 1973; 242: 190-191.

5 Mansfield P, Grannell PK. NMR 'diffraction' in solids? J Phys C Solid State Phys 1973; 6: L422-L426.

6 Kreis R, Boesch C. Liquid-crystal-like structures of human muscle demonstrated by in vivo observation of direct dipolar coupling in localized proton magnetic resonance spectroscopy. J Magn Reson B 1994; 104: 189-192.

7 Krssak M, Falk Petersen K, Dresner A, Di Pietro L, Vogel SM, Rothman DL et al. Intramyocellular lipid concentrations are correlated with insulin sensitivity in humans: a 1H NMR spectroscopy study. Diabetologia 1999; 42: 113-116.

8 Rico-Sanz J, Hajnal JV, Thomas EL, Mierisová S, Ala-Korpela M, Bell JD. Intracellular and extracellular skeletal muscle triglyceride metabolism during alternating intensity exercise in humans. J Physiol 1998; 510(Pt 2): 615-622.

9 Oliveira Junior SA, Padovani CR, Rodrigues SA, Silva NR, Martinez PF, Campos DH et al. Extensive impact of saturated fatty acids on metabolic and cardiovascular profile in rats with diet-induced obesity: a canonical analysis. Cardiovasc Diabetol 2013; 12: 65.

10 Kang JX, Li Y, Leaf A. Regulation of sodium channel gene expression by class I antiarrhythmic drugs and n-3 polyunsaturated fatty acids in cultured neonatal rat cardiac myocytes. Proc Natl Acad Sci USA 1997; 94: 2724-2788.

11 Seidelin KN. Fatty aid composition of adipose tissue in human. Implications for the dietary fat-serum cholesterol-CHD issue. Prog Lipid Res 1995; 34: 199-217. 
12 Insull W, Lang PD, Hsi B, Yoshimura SJ. Studies of arteriosclerosis in Japanese and American men: I. Comparison of fatty acid composition of adipose tissue. J Clin Invest 1969; 48: 1313-1327.

13 Ren J, Dimitrov I, Sherry AD, Malloy CR. Composition of adipose tissue and marrow fat in humans by ${ }^{1} \mathrm{H}$ NMR at 7 tesla. J Lipid Res 2008; 49: 2055-2062.

14 Strobel K, van den Hoff J, Pietzsch J. Localized proton magnetic resonance spectroscopy of lipids in adipose tissue at high spatial resolution in mice in vivo. J Lipid Res 2008; 49: 473-480.

15 Lee Y, Jee HJ, Noh H, Kang GH, Park J, Cho J et al. In vivo (1) H-MRS hepatic lipid profiling in nonalcoholic fatty liver disease: an animal study at 9.4 T. Magn Reson Med 2013; 70: 620-629.

16 Burt CT, Glonek T, Bárány M. Analysis of phosphate metabolites, the intracellular $\mathrm{pH}$, and the state of adenosine triphosphate in intact muscle by phosphorus nuclear magnetic resonance. J Biol Chem 1976; 251: 2584-2591.

17 Gadian DG, Hoult DI, Radda GK, Seeley PJ, Chance B, Barlow C. Phosphorus nuclear magnetic resonance studies on normoxic and ischemic cardiac tissue. Proc Natl Acad Sci U S A 1976; 73: 4446-4448.

18 Radda GK, Bore PJ, Gadian DG, Ross BD, Styles P, Taylor DJ et al. 31P NMR examination of two patients with $\mathrm{NADH}-\mathrm{COQ}$ reductase deficiency. Nature 1982; 295: 608-609.

19 Szendroedi J, Chmelik M, Schmid AI, Nowotny P, Brehm A, Krssak M et al. Abnormal hepatic energy homeostasis in type 2 diabetes. Hepatology 2009; 50: 1079-1086.

20 Petersen KF, Dufour S, Befroy D, Garcia R, Shulman GI. Impaired mitochondrial activity in the insulin-resistant offspring of patients with type 2 diabetes. N Engl J Med 2004; 350: 664-671.

21 Petersen KF, Befroy D, Dufour S, Dziura J, Ariyan C, Rothman DL et al. Mitochondrial dysfunction in the elderly: possible role in insulin resistance. Science 2003; 300: 1140-1142.

22 Schmid AI, Szendroedi J, Chmelik M, Krssák M, Moser E, Roden M. Liver ATP synthesis is lower and relates to insulin sensitivity in patients with type 2 diabetes. Diabetes Care 2011; 34: 448-453.

23 Phielix E, Szendroedi J, Roden M. Mitochondrial function and insulin resistance during aging: a mini-review. Gerontology 2011; 57: 387-396.

24 Choi CS, Befroy DE, Codella R, Kim S, Reznick RM, Hwang YJ et al. Paradoxical effects of increased expression of PGC-1alpha on muscle mitochondrial function and insulin-stimulated muscle glucose metabolism. Proc Natl Acad Sci USA 2008; 105: 19926-19931.

25 Laurent D, Yerby B, Deacon R, Gao J. Diet-induced modulation of mitochondrial activity in rat muscle. J Am J Physiol Endocrinol Metab 2007; 293: E1169-E1177.

26 Lu M, Zhu XH, Zhang Y, Chen W. Intracellular redox state revealed by in vivo $31 \mathrm{P}$ MRS measurement of NAD+ and $\mathrm{NADH}$ contents in brains. Magn Reson Med 2014; 71: 1959-1972.

27 Laufs A, Livingstone R, Nowotny B, Nowotny P, Wickrath F, Giani G et al. Quantitative liver 31P magnetic resonance spectroscopy at 3T on a clinical scanner. Magn Reson Med 2014; 71: 1670-1675.

28 Shulman GI, Rothman DL, Jue T, Stein P, DeFronzo RA, Shulman RG. Quantitation of muscle glycogen synthesis in normal subjects and subjects with non-insulin-dependent diabetes by $13 \mathrm{C}$ nuclear magnetic resonance spectroscopy. N Engl J Med 1990; 322: 223-228.

29 Rothman DL, Shulman RG, Shulman GI. 31P nuclear magnetic resonance measurements of muscle glucose-6-phosphate. Evidence for reduced insulin-dependent muscle glucose transport or phosphorylation activity in non-insulin-dependent diabetes mellitus. J Clin Invest 1992; 89: 1069-1075.

30 Cline GW, Petersen KF, Krssak M, Shen J, Hundal RS, Trajanoski Z et al. Impaired glucose transport as a cause of decreased insulin-stimulated muscle glycogen synthesis in type 2 diabetes. N Engl J Med 1999; 341 240-246.
31 Barnes PR, Kemp GJ, Taylor DJ, Radda GK. Skeletal muscle metabolism in myotonic dystrophy: a 31P magnetic resonance spectroscopy study. Brain 1997; 120(Pt 10): 1699-1711.

32 Kemp GJ, Taylor DJ, Thompson CH, Hands LJ, Rajagopalan B, Styles P et al. Quantitative analysis by 31P magnetic resonance spectroscopy of abnormal mitochondrial oxidation in skeletal muscle during recovery from exercise. NMR Biomed 1993; 6: 302-310.

33 Spencer RG, Balschi JA, Leigh JS Jr, Ingwall JS. ATP synthesis and degradation rates in the perfused rat heart. 31P-nuclear magnetic resonance double saturation transfer measurements. Biophys $J$ 1988; 54: 921-929.

34 Rossiter HB, Ward SA, Howe FA, Wood DM, Kowalchuk JM, Griffiths JR et al. Effects of dichloroacetate on V02 and intramuscular 31P metabolite kinetics during high-intensity exercise in humans. J App/ Physiol 2003; 95: $1105-1115$.

$35 \mathrm{Du} F$, Zhu XH, Qiao H, Zhang X, Chen W. Efficient in vivo 31P magnetization transfer approach for noninvasively determining multiple kinetic parameters and metabolic fluxes of ATP metabolism in the human brain. Magn Reson Med 2007; 57: 103-114.

36 Hwang J-H, Perseghin G, Rothman DL, Cline GW, Magnusson I, Petersen KF et al. Impaired net hepatic glycogen synthesis in insulindependent diabetic subjects during mixed meal ingestion. A $13 \mathrm{C}$ nuclear magnetic resonance spectroscopy study. J Clin Invest 1995; 95: 783-787.

37 Kishore P, Gabriely I, Cui MH, Di Vito J, Gajavelli S, Hwang J-H et al. Role of hepatic glycogen breakdown in defective counterregulation of hypoglycemia in intensively treated type 1 diabetes. Diabetes 2006; 55 : 659-666.

38 Roser W, Beckmann N, Wiesmann U, Seelig J. Absolute quantification of the hepatic glycogen content in a patient with glycogen storage disease by 13C magnetic resonance spectroscopy. Magn Reson Imaging 1996; 14: 1217-1220.

39 Roden M, Perseghin G, Petersen KF, Hwang J-H, Cline GW, Gerow K et al. The roles of insulin and glucagon in the regulation of hepatic glycogen synthesis and turnover in humans. J Clin Invest 1996; 97: 642-648.

40 Hwang J-H, Bluml S, Leaf A, Ross BD. In vivo characterization of fatty acids in human adipose tissue using natural abundance $1 \mathrm{H}$ decoupled $13 \mathrm{C}$ MRS at $1.5 \mathrm{~T}$ : clinical applications to dietary therapy. NMR Biomed 2003; 16 : 160-167.

41 Rothman DL, De Feyter HM, de Graaf RA, Mason GF, Behar KL. 13C MRS studies of neuroenergetics and neurotransmitter cycling in humans. NMR Biomed 2011; 24: 943-957.

42 Mishkovsky M, Comment A, Gruetter R. In vivo detection of brain Krebs cycle intermediate by hyperpolarized magnetic resonance. J Cereb Blood Flow Metab 2012; 32: 2108-2113.

43 Choi C, Ganji SK, DeBerardinis RJ, Hatanpaa KJ, Rakheja D, Kovacs Z et al. 2-hydroxyglutarate detection by magnetic resonance spectroscopy in IDH-mutated patients with gliomas. Nat Med 2012; 18: 624-629.

This work is licensed under a Creative Commons Attribution-NonCommercial-ShareAlike 3.0 Unported License. The images or other third party material in this article are included in the article's Creative Commons license, unless indicated otherwise in the credit line; if the material is not included under the Creative Commons license, users will need to obtain permission from the license holder to reproduce the material. To view a copy of this license, visit http:// creativecommons.org/licenses/by-nc-sa/3.0/ 